\title{
Mapping Martian Atmospheric Pressure with Ground-Based Near Infrared Spectroscopy
}

\author{
Sarah A. Chamberlain ${ }^{\mathrm{A}, \mathrm{C}}$, Jeremy A. Bailey ${ }^{\mathrm{A}}$ and David Crisp ${ }^{\mathrm{B}}$ \\ ${ }^{\text {A }}$ Australian Centre for Astrobiology, Macquarie University, NSW 2109, Australia \\ B Jet Propulsion Laboratory/Caltech, 4800 Oak Grove Dr, Pasadena, CA 91125, USA \\ C Corresponding author. E-mail: schamber@els.mq.edu.au
}

Received 2005 August 24, accepted 2006 August 2

\begin{abstract}
We present images of Mars obtained in the $2.0 \mu \mathrm{m} \mathrm{CO}$ band with the United Kingdom Infrared Telescope (UKIRT). The images reveal topographic features of the Martian surface that are usually invisible in direct albedo images at visible or IR wavelengths, but agree remarkably well with the topography of the planet as mapped by the MOLA instrument on Mars Global Surveyor. The $\mathrm{CO}_{2}$ band depth is a measure of the absorbing column of $\mathrm{CO}_{2}$ and hence determined primarily by the surface atmospheric pressure. The surface pressure variations are detected with a sensitivity of 4-5 $\mathrm{Pa}$. We compare our data with radiative transfer models and find that the measured $\mathrm{CO}_{2}$ variation with pressure is consistent with the models. We discuss the possibility that similar observations might be used to observe atmospheric pressure changes due to Martian weather systems.
\end{abstract}

Keywords: planets and satellites: individual (Mars) — techniques: spectroscopic — infrared: solar system

\section{Introduction}

Images of Mars obtained with ground-based telescopes are normally dominated by surface albedo markings which have little correlation with the topography of the Martian surface. As a result, the major topographic features were not discovered until it was possible to view the planet close up using spacecraft. In particular, the major volcanoes, and the vast canyon system of Valles Marineris, were discovered by the Mariner 9 spacecraft in 1971. In hindsight it is sometimes possible to see the volcanoes as bright spots in good ground-based images, but it would not have been possible to infer their nature from such observations.

It is possible to study the topography of Mars by measuring the strength of $\mathrm{CO}_{2}$ absorption bands to give the $\mathrm{CO}_{2}$ column density, and hence the surface atmospheric pressure (since $\mathrm{CO}_{2}$ is the main atmospheric constituent). Several ground-based observations of this type were made in the 1960s and 1970s (Belton \& Hunten 1971; Woszczyk 1971; Parkinson \& Hunten 1973) using high spectral resolution observations of $\mathrm{CO}_{2}$ bands from $0.87 \mu \mathrm{m}$ to $1.05 \mu \mathrm{m}$. Although the mean surface pressure derived from these observations is approximately correct, the topographic results have poor spatial resolution and do not agree well with modern spacecraft data. In this paper we show that good quality maps of the topography of Mars can now be made with a ground-based telescope using the much stronger $\mathrm{CO}_{2}$ bands in the $2 \mu \mathrm{m}$ spectral region. The technique is essentially the same as that used by Bibring et al. (1991) with the ISM instrument on the Phobos 2 spacecraft to measure the topography in parts of the Martian tropical regions. While the topography of Mars is now, of course, well determined from spacecraft observations, these results do suggest a possible remote sensing approach to the measurement of surface pressure changes due to Martian weather systems.

\section{Observations}

Observations of Mars were obtained during the 2003 August opposition. The ground-based United Kingdom Infrared Telescope (UKIRT) was used with the UKIRT Imaging Spectrometer UIST, (Ramsay Howat et al. 1998) to obtain images and spectra at near-infrared wavelengths $(0.8-3.6 \mu \mathrm{m})$. The telescope was chosen because the high altitude Mauna Kea site, and the recent telescope upgrades project (Hawarden et al. 1998) provides excellent image quality and minimal terrestrial atmospheric absorption. The UKIRT telescope control system incorporates the "virtual guide telescope - active guiding' scheme (Bailey \& Prestage 1997), which allows precision offsetting of the telescope while continually locked to a guide star, with automatic compensation for the non-sidereal motion of Mars.

The spectroscopic observations were obtained with the spectrometer slit in a north-south orientation on the sky. The telescope was moved incrementally across the disk in the westward direction, recording a long-slit spectrum at each position. Scan increments of $0.25 \mathrm{arcsec}$ were used, taking $51 \mathrm{~min}$ to complete. Observations were taken with a spectral resolving power of 950 in the HK waveband (1.4-2.5 $\mu \mathrm{m})$, with seeing conditions as good 
Table 1. Observational parameters

\begin{tabular}{lcc}
\hline & 2003 Aug 17 & 2003 Sep 4 \\
\hline UT $^{-}$at start of scan & $10: 52: 48$ & $10: 04: 20$ \\
UT at finish of scan & $11: 43: 51$ & $10: 55: 17$ \\
Sub-Earth longitude $\left.{ }^{\circ}{ }^{\circ}\right]$ & 231.9 & 61 \\
Sub-Earth latitude $\left.{ }^{\circ}\right]$ & -19.31 & -18.95 \\
Angular diameter $[\operatorname{arcsec}]$ & 24.672 & 24.822 \\
Solar longitude $\left.L_{\mathrm{s}}{ }^{\circ}\right]$ & 242.9 & 254.3 \\
Airmass at start of scan & 1.232 & 1.236 \\
Airmass at finish of scan & 1.217 & 1.279 \\
Radial velocity of Mars at start of scan $\left[\mathrm{km} \mathrm{s}^{-1}\right]^{\mathrm{A}}$ & -2.45 & 1.98 \\
Radial velocity of Mars at finish of scan $\left[\mathrm{km} \mathrm{s}^{-1}\right]^{\mathrm{A}}$ & -2.34 & 2.08 \\
\hline
\end{tabular}

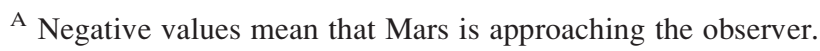

as 0.35 arcsec giving a spatial resolution of $97 \mathrm{~km}$ at the centre of the disk. Observational parameters for the observations taken on 2003 August 17 and 2003 September 4 are detailed in Table 1. Basic data reduction has been applied including flat fielding, sky subtraction, and wavelength calibration (using an argon lamp). The individual spectra were then assembled into a threedimensional 'data cube' with two spatial dimensions $(x, y)$ and a spectral dimension $(z)$. The accurate scanning provided by the UKIRT active guiding scheme made combining the slit spectra into the data cube straightforward. Spectra from any location on Mars can then be extracted by slicing the data cube across the spatial axes and images of Mars at any wavelength can be obtained by slicing across the spectral axis.

Figure 1 shows uncalibrated spectra from a high altitude position and a low altitude position. The absorption feature near $2 \mu \mathrm{m}$ is due to the combined effect of $\mathrm{CO}_{2}$ in the Martian atmosphere and the telluric $\mathrm{CO}_{2}$ column at our observing site. Since the Martian atmosphere is composed of $95.3 \% \mathrm{CO}_{2}$, the variations in the $2 \mu \mathrm{m}$ absorption feature are primarily due to the variations in atmospheric path length and therefore outline the topography of Mars. Topography can be observed in several $\mathrm{CO}_{2}$ bands in our wavelength region, but we find the $2 \mu \mathrm{m}$ band gives the best signal-to-noise ratio, despite being more saturated than other bands. A simple colour index can be created by taking the ratio of an image of Mars extracted from the data cube at wavelengths corresponding to the deepest region of the absorption band (2.001-2.018 $\mu \mathrm{m})$ with a similar image of Mars created at the wavelengths of the nearby continuum (1.981-1.996 $\mu \mathrm{m})$, Eqn (1).

$$
\mathrm{CO}_{2} \text { Index }=\frac{\sum \text { Flux }_{\text {absorption band wavelengths }}}{\sum \text { Flux }_{\text {continuum wavelengths }}}
$$

Figure 2, left, shows an infrared albedo image extracted from the data cube over the wavelength range of 2.2-2.3 $\mu \mathrm{m}$, the $\mathrm{CO}_{2}$ index map and the corresponding MOLA topographic map for observations taken on 2003 August 17 and September 4.

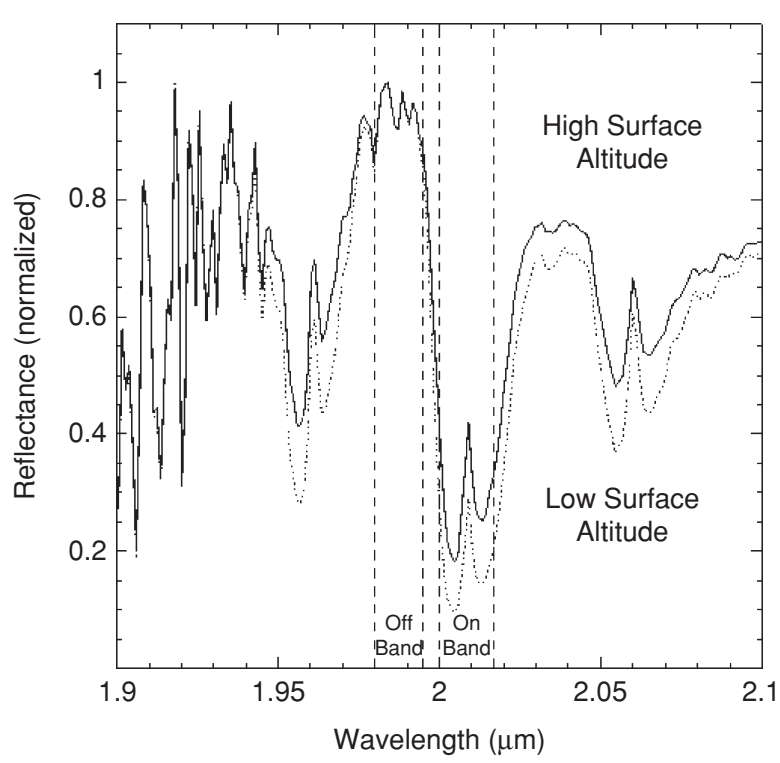

Figure 1 A comparison between the spectra of low-lying and higher altitude regions of Mars. The gaseous $\mathrm{CO}_{2}$ absorption feature at $2 \mu \mathrm{m}$ is shown to be deeper for regions of low altitude due to the larger path length of reflected light through the Martian atmosphere. The vertical lines indicate the wavelength regions (on and off the peak $2 \mu \mathrm{m}$ band) used for our $\mathrm{CO}_{2}$ band depth index.

The observations used here have not been flux calibrated or corrected for terrestrial atmospheric absorption. This is normally done by dividing the observed spectrum by the spectrum of a relatively featureless standard star observed under similar atmospheric conditions and thereby removing the common spectral component (absorption by the terrestrial atmosphere). This method cannot be used for Mars at these wavelengths, because $\mathrm{CO}_{2}$ absorption is present in both the Mars and Earth atmospheres at similar strengths and is composed of many saturated narrow lines (unresolved at the resolution of our spectra). Once a line is saturated it ceases to behave linearly. The terrestrial absorption will therefore produce a greater absorption in the spectra of the standard star, due to the initial lack of features, than in the Martian spectra which already have strong absorption at these wavelengths. This effect is 
(a)

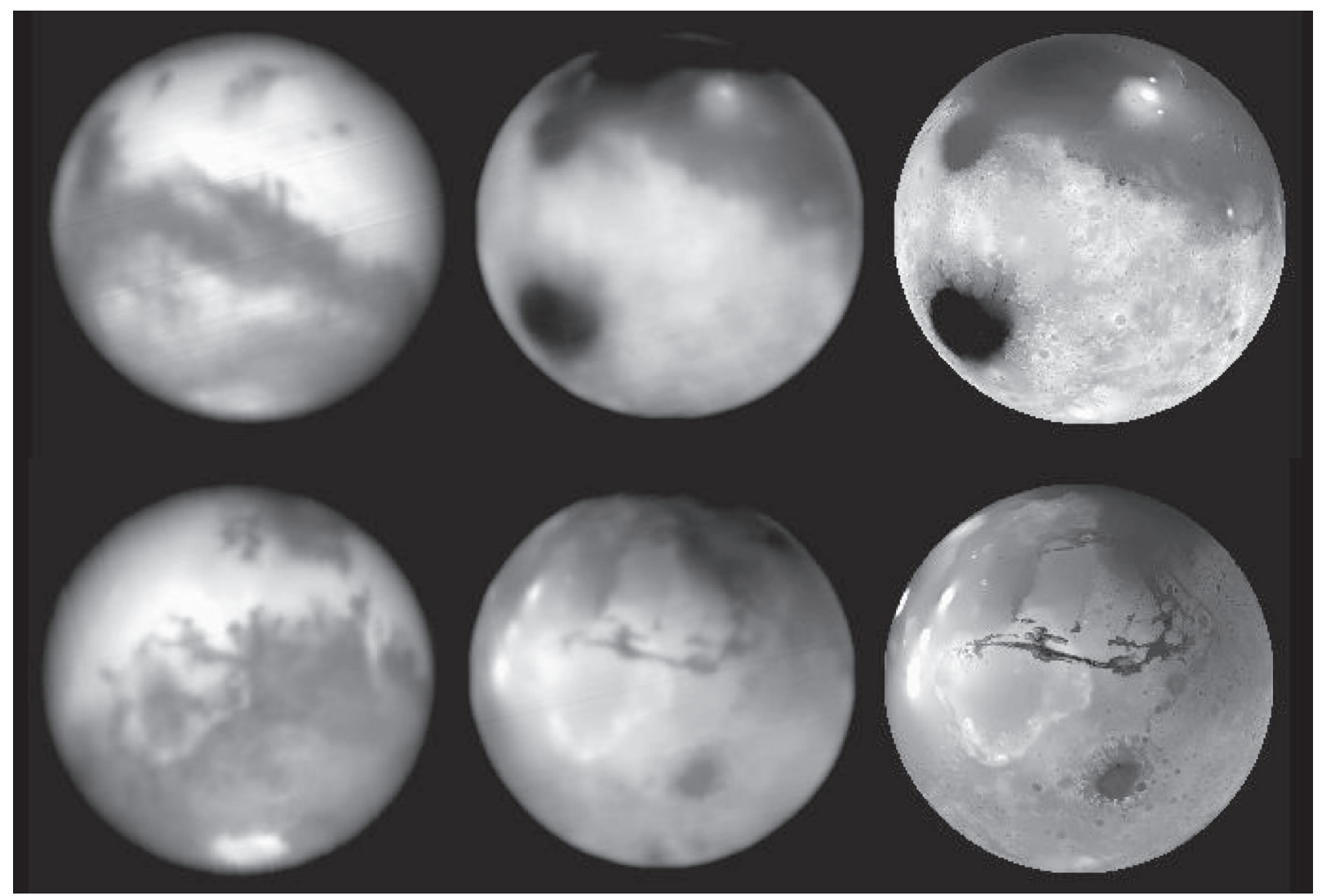

Figure 2 Images of Mars on (a) 2003 Aug 17 and (b) 2003 Sep 4. Left: An albedo image extracted from the data cube using 2.2-2.3 $\mu \mathrm{m}$ wavelengths. Centre: $\mathrm{A} \mathrm{CO}_{2}$ index image extracted from the data cube using wavelengths about the $2 \mu \mathrm{m}$ gaseous $\mathrm{CO}_{2}$ absorption band. Right: An orthographic projection of Mars Global Surveyor MOLA data, for a similar orientation as the observations. A slight distortion between the centre and right images is due to a significant rotation of Mars during the $51 \mathrm{~min}$ scan.

maximized at opposition due to the lack of relative velocity along the line of sight between the two planets, and would result in removing more than just the terrestrial component of the $\mathrm{CO}_{2}$ absorption. However, since the air mass over which Mars was observed changed little during the scans, the terrestrial $\mathrm{CO}_{2}$ absorption is nearly constant, and all the variations observed in our images are due to $\mathrm{CO}_{2}$ in the Mars atmosphere. The effects of the Earth's atmosphere can be dealt with by the modelling procedure described later.

\section{Discussion}

It is clear on comparing the albedo and the $\mathrm{CO}_{2}$ index images in Figure 2, that the albedo images show very little correspondence with the actual topography. The $\mathrm{CO}_{2}$ index image clearly outlines the surface dichotomy between the older southern highlands and the younger northern lowlands. This is especially visible in the August images (Figure 2a), as the dark low-lying region of the Hellas Planitia is outlined by the bright southern highlands and the volcano Elysium Mons appears as a bright feature compared to the surrounding dark northern lowlands. Topographic features that were first discovered by orbiting spacecraft can also be seen in the ground-based $\mathrm{CO}_{2}$ index images, such as Appollinaris
Patera (Figure 2a), Valles Marineris, and Argyre Planitia (Figure 2b).

The $\mathrm{CO}_{2}$ index maps show a good correlation to maps produced by the Mars Orbiting Laser Altimeter (MOLA) on board the Mars Global Surveyor (MGS). MOLA obtained topographic data of Mars by calculating the travel time of a laser pulse from the orbiter to the Martian surface, providing a vertical resolution of $2 \mathrm{~m}$ and a horizontal resolution of $160 \mathrm{~m}$ (Smith et al. 1998). This provided a more accurate topographic map than can be produced through atmospheric band strength comparisons from Earth. On comparison with MOLA maps, every sufficiently large feature can be identified in the ground-based $\mathrm{CO}_{2}$ index image, such as the smaller volcanoes that appear as bright spots above the Tharsis Montes (Figure 2b) and details in the cratering around Argyre Planitia and the smaller scale crustal fissures associated with (west) Valles Marineris are also visible (Figure 2b). Although the $\mathrm{CO}_{2}$ index image shows topographic details, it is unreliable at the south pole where $\mathrm{CO}_{2}$ ice absorption is strong in the $2 \mu \mathrm{m}$ region, and in the far north where the polar hood clouds are present. These effects can be seen as darkened regions about the poles that do not correspond to similar regions on the MOLA images. 
Table 2. Measured $\mathrm{CO}_{2}$ index values ${ }^{\mathrm{A}}$

\begin{tabular}{cccccc}
\hline & $\begin{array}{c}\text { Martian latitude } \\
{\left[{ }^{\circ}\right]}\end{array}$ & $\begin{array}{c}\text { Martian longitude } \\
{\left[{ }^{\circ}\right]}\end{array}$ & $\begin{array}{c}\text { Surface pressure } \\
{[\mathrm{Pa}]}\end{array}$ & $\begin{array}{c}\text { Mean } \mathrm{CO}_{2} \\
\text { index value }\end{array}$ & $\begin{array}{c}\text { Sigma over } \\
9 \text { pixels }\left[\times 10^{-3}\right]\end{array}$ \\
\hline 2003 Aug 17 & 135.0 & 10.0 & 792 & 0.2454 & 1.290 \\
& $\mathbf{1 1 0 . 0}$ & $\mathbf{- 1 2 . 5}$ & $\mathbf{5 4 5}$ & $\mathbf{0 . 3 5 0 4}$ & $\mathbf{1 . 5 6 0}$ \\
& $\mathbf{1 1 3 . 0}$ & $\mathbf{- 2 2 . 5}$ & $\mathbf{5 3 3}$ & $\mathbf{0 . 3 5 8 2}$ & $\mathbf{1 . 0 5 7}$ \\
& 120.0 & -37.0 & 569 & 0.3397 & 1.796 \\
& 106.0 & 3.0 & 669 & 0.2994 & 4.632 \\
& $\mathbf{1 1 0 . 0}$ & $\mathbf{- 1 5 . 0}$ & $\mathbf{5 3 8}$ & $\mathbf{0 . 3 5 3 3}$ & $\mathbf{1 . 5 0 9}$ \\
& -42.0 & -22.0 & 557 & 0.3484 & 0.717 \\
& -90.0 & -28.0 & 458 & 0.3832 & 1.730 \\
& -65.0 & -35.0 & 482 & 0.3724 & 3.956 \\
& -82.5 & -15.0 & 442 & 0.3882 & 2.196 \\
& -50.0 & -32.5 & 560 & 0.3527 & 1.585 \\
& $\mathbf{- 6 4 . 0}$ & $\mathbf{- 2 5}$ & $\mathbf{4 6 7}$ & $\mathbf{0 . 3 8 3 0}$ & $\mathbf{1 . 2 9 5}$ \\
& $\mathbf{- 7 7 . 0}$ & $\mathbf{- 2 2 . 5}$ & $\mathbf{4 9 9}$ & $\mathbf{0 . 3 7 4 0}$ & $\mathbf{1 . 7 6 3}$ \\
\hline
\end{tabular}

${ }^{A}$ Values in bold were taken closer to the centre of the observed Martian disk.

We have estimated the sensitivity of our observations to surface atmospheric pressure as follows. We selected regions on our $\mathrm{CO}_{2}$ images where there was little surface topographic structure and measured the mean and standard deviation of the $\mathrm{CO}_{2}$ index values over these regions (Table 2). We then used the European Mars Climate Database (Lewis et al. 1999) to obtain the expected surface pressure at these regions for the solar longitude angle $L_{\mathrm{s}}$ of our observations and used these to derive a rough calibration of $\mathrm{CO}_{2}$ index against pressure. We can then convert the $\mathrm{CO}_{2}$ index standard deviation to a pressure sensitivity, which we find to be $\sim 4-5 \mathrm{~Pa}$ (0.04-0.05 mbar). This could, in principle, be improved by using a larger telescope, by using a wider range of $\mathrm{CO}_{2}$ bands, and by using a more efficient observing system (to avoid saturation with UIST we had to use a narrower-than-optimal slit, and the shortest possible exposure time, which led to less than $30 \%$ of time being spent integrating). Thus pressure sensitivity down to $\sim 1$ Pa may be possible in the future.

Analysis of the pressure measurements by the Viking landers showed pressure changes on a range of timescales. Seasonal variations are caused by the $\mathrm{CO}_{2}$ exchange between the polar caps and the atmosphere. Pressure changes on timescales of a few days with amplitudes of around $50 \mathrm{~Pa}$ (in a total pressure of $\sim 800 \mathrm{~Pa}$ ) have been identified as baroclinic waves (Barnes 1981; Collins et al. 1996). Diurnal pressure variations due to strong thermal tides are also observed.

These surface pressure effects should be present in $\mathrm{CO}_{2}$ index images of the type we have obtained, and could, in principle, be detected by this type of observation. However, there are several complications. $\mathrm{CO}_{2}$ band strength is determined not just by surface pressure, but also by both the effects of dust in the Martian atmosphere and by temperature, which change the distribution of line strengths within each $\mathrm{CO}_{2}$ band, and hence the line shape.

\subsection{Modelling}

To further investigate these effects we have used the radiative transfer model SMART (spectral mapping atmospheric radiative transfer) to simulate spectra from Mars as seen through the Earth's atmosphere. SMART uses a line-by-line model for the molecular absorption and uses the DISORT package (Stamnes et al. 1988), to solve the radiative transfer equations using the discrete ordinate method. The detailed physics incorporated in SMART is described by Meadows and Crisp (1996). The simulated spectra were produced by running SMART twice; the first run modelled the passage of sunlight through the Martian atmosphere and the resulting high-resolution spectrum was used as the input for a terrestrial model for the second run to model the effects of terrestrial atmospheric absorption. The final spectrum was then used to determine a $\mathrm{CO}_{2}$ index for comparison with the observed data. This approach is essential to correctly handle the terrestrial atmospheric absorption.

The models use molecular absorption line data taken from the HITRAN 2000 database (Rothman et al. 2003). The Mars model includes absorption from $\mathrm{CO}_{2}$, $\mathrm{CO}, \mathrm{H}_{2} \mathrm{O}, \mathrm{O}_{2}$, and $\mathrm{O}_{3}$. The Earth atmosphere transmission model uses the atmospheric profile for the ICRCCM (Intercomparison of Radiative Codes in Climate Models) mid-latitude summer atmosphere and includes absorption from $\mathrm{CO}_{2}, \mathrm{H}_{2} \mathrm{O}, \mathrm{O}_{2}, \mathrm{CH}_{4}, \mathrm{CO}, \mathrm{O}_{3}$, and $\mathrm{N}_{2} \mathrm{O}$.

A range of models were calculated to investigate the effect on $\mathrm{CO}_{2}$ index of the Martian surface temperature, surface pressure and dust optical depth. The different surface temperature models (Figure 3a) use temperature profiles for different regions of Mars taken from the European Mars Climate Database (Lewis et al. 1999) for the $L_{\mathrm{s}}$ of our observations. They all use a surface pressure of $600 \mathrm{~Pa}$ and a dust optical depth of $\tau=0.6$ 


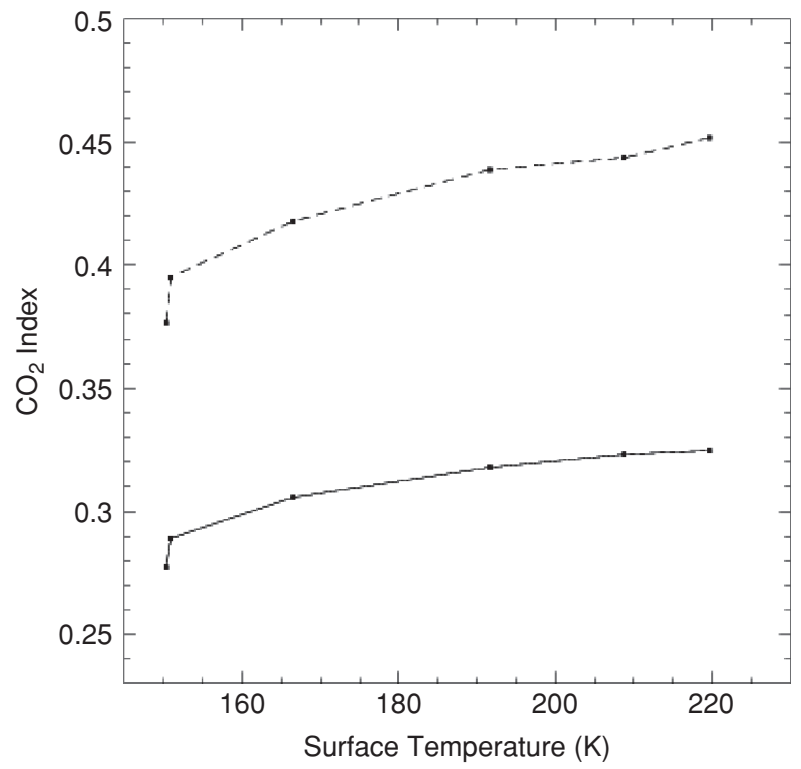

(a)

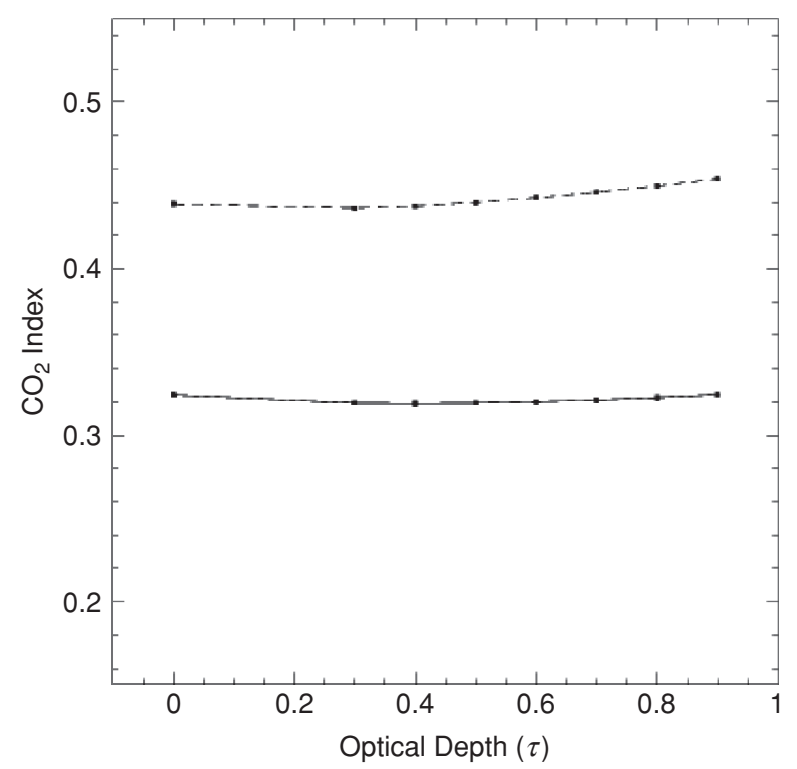

(b)

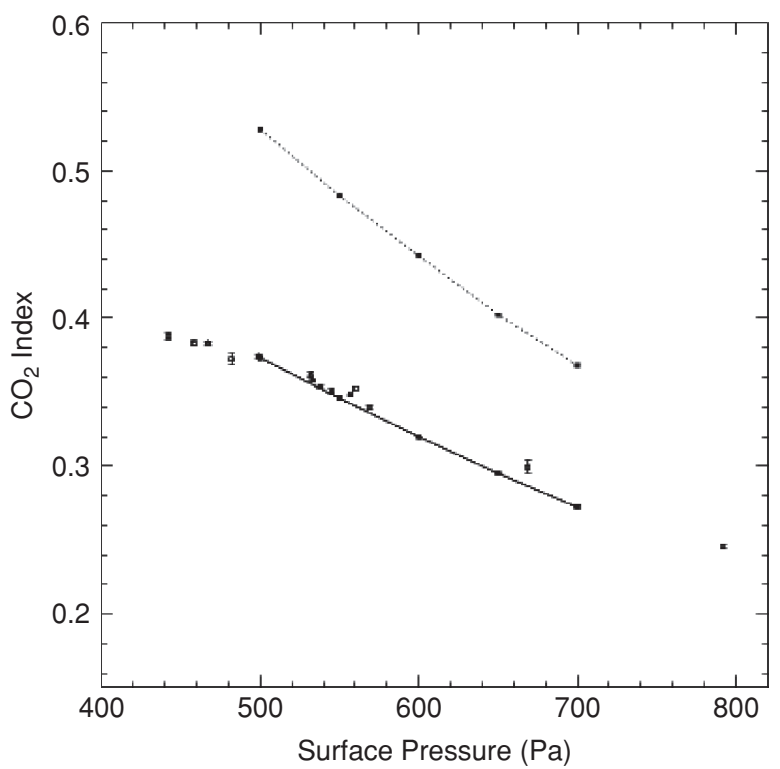

(c)

Figure 3 (a) Variation in the modelled $2 \mu \mathrm{m} \mathrm{CO}_{2}$ index as a function of Martian surface temperature. The dashed line shows the value before passing through the Earth's atmosphere (i.e. as would be seen by a space instrument) and the solid line shows the value as seen through the modelled terrestrial atmosphere (i.e. as seen by a ground-based telescope). (b) Variation in the modelled $2 \mu \mathrm{m} \mathrm{CO}_{2}$ index for different dust optical depths. The dashed line shows the value as seen from space and the solid line shows the value as seen through the terrestrial atmosphere. (c) Variation in the modelled $2 \mu \mathrm{m} \mathrm{CO}$ index as a function of atmospheric pressure. The dotted line shows the value seen from space with a dust optical depth of 0.6 and the solid line shows the value as seen through the terrestrial atmosphere. The points show measured values taken from our Mars observations in 2003, points indicated by hollow triangles were obtained within $15^{\circ}$ of the Martian disk's observed centre, points indicated by hollow squares are taken from points between $30^{\circ}$ and $15^{\circ}$ of the Martian disk's observed centre.

(at $600 \mathrm{~nm}$ ). The other models all have a surface temperature of $210 \mathrm{~K}$ and vary the dust optical depth (Figure $3 b$ ) or surface pressure (Figure 3c). In Figure $3 \mathrm{c}$ the model results are also compared with our measured $\mathrm{CO}_{2}$ index values as a function of pressure, from the data in Table 2. An optical depth of 0.6 was chosen for the model, which is in good agreement with the optical depths obtained by the MGS TES (thermal emission spectrometer). Visible optical depths are approximately two times larger than the TES thermal IR optical depths (Lemmon et al. 2004). TES results measured optical densities of 0.28 (0.56 at visible wavelengths) and 0.25 (0.5 at visible wavelengths) for solar longitudes of $242.9^{\circ}$ and $254.3^{\circ}$ respectively (Smith 2006).

From Figure 3 it is apparent that observing through the $\mathrm{CO}_{2}$ absorption bands in the Earth's atmosphere leads to stronger $\mathrm{CO}_{2}$ bands (smaller $\mathrm{CO}_{2}$ indices), as expected, and also reduces the sensitivity of $\mathrm{CO}_{2}$ index 
to changes in the atmospheric properties. Nevertheless our $\mathrm{CO}_{2}$ index still shows substantial variation with pressure, some variation with temperature, and little variation with dust.

Our observed variation of $\mathrm{CO}_{2}$ index with pressure is in good agreement with the modelled $\mathrm{CO}_{2}$ index variations with pressure. Differences seen in the scatter in the observations are likely the result of the changing Martian airmass at different surface locations, however we have chosen points near the centre of the disk to minimize this effect. The variations contributed by the dust-related optical depths seem to be suppressed by the terrestrial atmosphere resulting in a near-trivial contribution to the overall $\mathrm{CO}_{2}$ index of Mars as seen from ground-based observations.

The results also show that separating the effects of pressure, temperature, and dust is not going to be possible from the measurement of a single band-strength index as we are using here, since measurements of pressure change could be masked or strongly biased by changes in gas temperature or dust optical depth. However, higher resolution observations that are able to resolve the individual lines within the $\mathrm{CO}_{2}$ bands, could provide sufficient information to separate the various effects, by comparing weak lines, which will be linear in pressure, to strong saturated lines which will be most sensitive to dust. Several IR instruments on groundbased telescopes can provide sufficient resolving power ( $R=\lambda / \Delta \lambda \approx 20000$ ) to resolve the lines, whereas current spacecraft instruments do not have sufficient resolution.

Measurements of the surface pressure distribution on Mars are of great interest as they provide the key data needed for testing of, and assimilation into, General Circulation Models (GCMs) of the Martian atmosphere (Forget et al. 1999). Until a network of surface stations can be established on Mars, remote-sensing techniques will provide the only way of obtaining such data. We suggest that ground-based IR spectroscopy can play a useful role in developing such techniques.

\section{Conclusion}

These results show that the atmospheric pressure changes due to topography can be easily observed by a simple comparison of on and off band images in the $2 \mu \mathrm{m} \mathrm{CO} \mathrm{CO}_{2}$ band. The observations are in good correspondence with MOLA/MGS data taken from Martian orbit. Surface pressure changes are detected with a sensitivity of $\sim 4-5 \mathrm{~Pa}$. Radiative transfer modelling shows that the observed $\mathrm{CO}_{2}$ band variation with pressure is in good agreement with modelled $\mathrm{CO}_{2}$ band variations. Similar techniques might be used in the future to detect and study atmospheric pressure changes due to Martian weather systems.

\section{Acknowledgements}

We thank the staff of the Joint Astronomy Centre, in particular Paul Hirst and Thor Wold, our support scientist and telescope operator, during our UKIRT observations of Mars. We also thank the NASA Astrobiology Institute's Virtual Planetary Laboratory Lead Team (PI Vikki Meadows), who provided financial support for the observing run. The NAI's Virtual Planetary Laboratory is supported by the National Aeronautics and Space Administration through the NASA Astrobiology Institute under Cooperative Agreement No. CAN-00-OSS-01. The Australian Centre for Astrobiology is supported by the Macquarie University Biotechnology Research Institute.

\section{References}

Bailey, J. \& Prestage, R. P. 1997, SPIE Proc, 3112, 124

Barnes, J. R. 1981, JAtS, 38, 225

Belton, M. J. S. \& Hunten, D. M. 1971, Icar, 15, 204

Bibring, J. P. et al. 1991, P\&SS, 39, 225

Collins, M., Lewis, S. R., Read, P. L. \& Hourdin, F. 1996, Icar, 120,344

Forget, F. et al. 1999, JGRE, 104, 24155

Hawarden, T. G. et al. 1998, SPIE Proc, 3352, 52

Lemmon, M. T. et al. 2004, Sci, 306, 1753

Lewis, S. R. et al. 1999, JGRE, 104, 24177

Meadows, V. S. \& Crisp, D. 1996, JGRE, 101, 4595

Parkinson, T. D. \& Hunten, D. M. 1973, Icar, 18, 29

Ramsay Howat, S. K. et al. 1998, SPIE Proc, 3354, 456

Rothman, L. S. et al. 2003, JQSRT, 82, 5

Smith, D. E. et al. 1998, Sci, 279, 1686

Smith, M. D. 2006, Second workshop on Mars atmospheric modelling and observations proceedings, Eds Forget, F., Lopez-Valverde, M. A., Desjean, M. C., Huot, J. P., Lefevre, F., Lebonnois, S., Lewis, S. R., Millour, E., Read, P. L. \& Wilson, R. J. (LMD, IAA, AOPP, CNES, ESA), 211

Smith, M. D. et al. 2003, JGRE, 108, 5115

Stamnes, K. et al. 1988, ApOpt, 27, 2502

Woszczyk, A. 1971, in IAU Symp., 40, Planetary Atmospheres, Eds Sagan, C. Owen, T. C. \& Smith, H. J. (Dordrecht: Reidel), 203 